\title{
Amide bonds assemble pili on the surface of bacilli
}

\author{
Jonathan M. Budzik ${ }^{\dagger}$, Luciano A. Marraffini ${ }^{\dagger}$, Puneet Souda ${ }^{\ddagger}$, Julian P. Whitelegge ${ }^{\ddagger}$, Kym F. Faull ${ }^{\ddagger}$, \\ and Olaf Schneewind ${ }^{\dagger \S}$ \\ †Department of Microbiology, University of Chicago, 920 East 58th Street, Chicago, IL 60637; and ¥Pasarow Mass Spectrometry Laboratory, Neuropsychiatric \\ Institute, Semel Institute for Neuroscience and Human Behavior, David Geffen School of Medicine, University of California, Los Angeles, CA 90095
}

Edited by Robert Haselkorn, University of Chicago, Chicago, IL, and approved May 8, 2008 (received for review April 11, 2008)

Pilin precursors are the building blocks of pili on the surface of Gram-positive bacteria; however, the assembly mechanisms of these adhesive fibers are unknown. Here, we describe the chemical bonds that assemble BcpA pilin subunits on the surface of Bacillus cereus. Sortase D cleaves BcpA precursor between the threonine (T) and the glycine (G) residues of its LPXTG sorting signal and catalyzes formation of an amide bond between threonine $(T)$ of the sorting signal and lysine (K) in the YPKN motif of another BcpA subunit. Three CNA B domains of BcpA generate intramolecular amide bonds, and one of these contributes also to pilus formation. Conservation of catalysts and structural elements in pilin precursors in Gram-positive bacteria suggests a universal mechanism of fiber assembly.

CNA B domain | LPXTG sorting signal | sortase | YPKN motif

B acteria elaborate adhesive pili that promote their attachment and invasion of tissues (1-5). Host immunization with pilin subunits can elicit antibodies that protect against bacterial infections, suggesting that pilins may be suitable vaccines to prevent pneumonia and neonatal meningitis $(2,6,7)$. Pili are immobilized in the cell-wall envelope of Gram-positive bacteria (8). Sortases cleave the sorting signal of surface proteins and pilin proteins to form amide bonds between their C-terminal carboxyl group and the amino group of peptidoglycan crossbridges in the cell wall $(9,10)$. Assembly of pili in Corynebacterium diphtheriae and other Gram-positive bacteria also requires sortase genes and the LPXTG sorting signal and YPKN motif of a major pilin protein $(8,11)$. Minor pilin proteins harbor sorting signals but lack the YPKN pilin motif (8).

Bacillus cereus forms pili, 1- to 5- $\mu$ m-long fibers, on its cell surface, and the genes for pilus assembly reside in the $b c p A$ $s r t D-b c p B$ operon (12). BcpA, the major pilin, is distributed throughout the pilus fiber, whereas the minor pilin, $\mathrm{BcpB}$, is positioned at its tip (12). Sortase D (srtD) is required for the formation of pili, which depends also on the YPKN motif and LPXTG sorting signal of BcpA (12). BcpB is dispensable for the formation of BcpA pili (12). Previous work proposed that sortase may cleave the sorting signal of the pilin precursor to form a bond with another pilin subunit, perhaps involving the YPKN motif $(8,13)$. In agreement with this, substitution of lysine, carrying a side chain $\varepsilon$-amino group, with alanine in the YPKN motif of $\mathrm{BcpA}$, abolishes pilus formation in B. cereus (12). Sortase A, the transpeptidase that immobilizes surface proteins in the cell wall envelope $(9,14)$, is required for deposition of $\mathrm{BcpA}$ pili in the bacterial envelope but is otherwise dispensable for fiber formation (12).

$\mathrm{X}$-ray crystallography revealed the 3D structure of recombinant pilin precursors from Streptococcus pyogenes and Streptococcus agalactiae $(15,16)$. The Ig-like fold of CNA B domains (17) in pilin precursors harbor amide bonds between lysine and asparagine, generated by a catalytic mechanism that involves a conserved glutamic acid residue with hydrogen bonding to amide $\mathrm{C}=\mathrm{O}$ and $\mathrm{NH}$ groups (15). However, CNA B domains form intramolecular amide (isopeptide) bonds that cannot link multiple pilin subunits. A possible contribution of CNA B domain- mediated amide bond formation toward pilus formation is not yet known.

\section{Results}

Assembly of Engineered BcpA Pili. Bacillus anthracis, a member of the $B$. cereus group, was transformed with pJB39, a plasmid that encodes $b c p A-s r t D$ under control of the IPTG inducible $\mathrm{P}_{\text {spac }}$ promoter (12) (Fig. 1A). Formation of BcpA pili was revealed by electron microscopy and ImmunoGold labeling with BcpA antibodies on the surface of bacilli harboring pJB39 but not the control plasmid pLM5 (Fig. 1B). Assembled pilus fibers are high-molecular-weight macromolecules $\left(\mathrm{BcpA}_{\mathrm{HMw}}\right)$ that resist extraction with hot SDS and can be detected by immunoblotting with BcpA-specific antibodies (Fig. 1B). Alanine substitution of Lys $^{162}$ (pJB57), but not of $\mathrm{Asn}^{163}$ (pJB112), abolished pilus formation, documenting the requirement of Lys $^{162}$ within the YPKN motif for pilus assembly (Fig. $1 B$ and $C$ ). Expression of bcp $A$-srtD in sortase A (srt $A)$ mutant strains triggers the release of assembled pili into the extracellular medium (12) (Fig. 1C). To purify pili, a Met-His 6 peptide was inserted near the YPKN motif of BcpA. This insertion did not affect pilus assembly (Fig. $1 \mathrm{~A}$, pJB103).

Purification and Analysis of BcpA Pili. After signal peptide and sorting signal removal, the mature form of the BcpA variant encoded by pJB103 encompasses four methionine residues. Cyanogen bromide $(\mathrm{CNBr})$, a chemical that cleaves after methionine, was predicted to cut BcpA into five fragments $\left(M_{\mathrm{r}}\right.$ 7,680.61, 5,954.60, 2,751.02, 38,848.22, and 428.53). The His affinity tag resides in the third fragment and allows convenient purification by affinity chromatography on Ni-NTA Sepharose. B. anthracis $\operatorname{srtA}$ (pJB103) was grown on agar, suspended in water, and centrifuged to separate pili from the bacterial sediment. $\mathrm{CNBr}$ cleavage products of BcpA pili were purified by Ni-NTA chromatography (Fig. 1D) and RP-HPLC (Fig. 2A). Three predominant compounds with increasing molecular mass, designated A $(m / z 10,835.45)$ (Fig. $2 B), \mathrm{B}(\mathrm{m} / \mathrm{z} 16,796.52)$, and $\mathrm{C}(>60 \mathrm{kDa})$ were identified by MALDI-MS (Fig. $2 A$ Inset). All three $\mathrm{CNBr}$ cleavage products reacted with $\mathrm{BcpA}$ antibodies and bound to His-probe (Fig. $2 A$, Inset). Nevertheless, their molecular mass could not be explained by $\mathrm{CNBr}$ cleavage of $\mathrm{BcpA}$ alone and must be accounted for by chemical bonds that form during assembly of pilus fibers.

Edman degradation of compound A released three amino acid residues per cycle [Fig. $2 B$, identified residues printed in bold;

Author contributions: J.M.B., L.A.M., K.F.F., and O.S. designed research; J.M.B. and J.P.W. performed research; J.M.B., L.A.M., and P.S. contributed new reagents/analytic tools; J.M.B., L.A.M., J.P.W., K.F.F., and O.S. analyzed data; and J.M.B., K.F.F., and O.S. wrote the paper.

The authors declare no conflict of interest.

This article is a PNAS Direct Submission.

§To whom correspondence should be addressed. E-mail: oschnee@bsd.uchicago.edu.

This article contains supporting information online at www.pnas.org/cgi/content/full/ 0803565105/DCSupplemental.

C 2008 by The National Academy of Sciences of the USA 
A

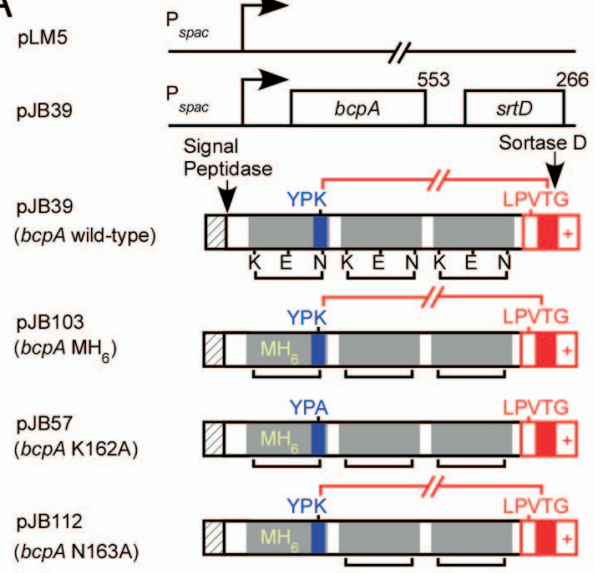

B

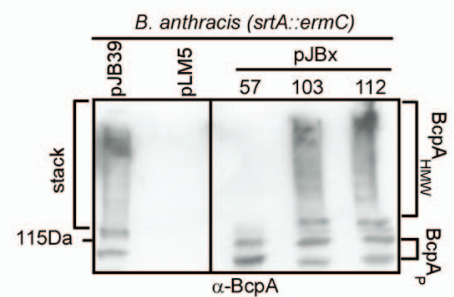

D

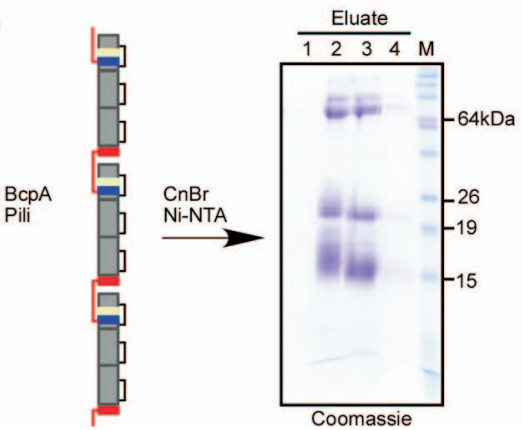

C

B. anthracis (srtA::ermC) Culture medium

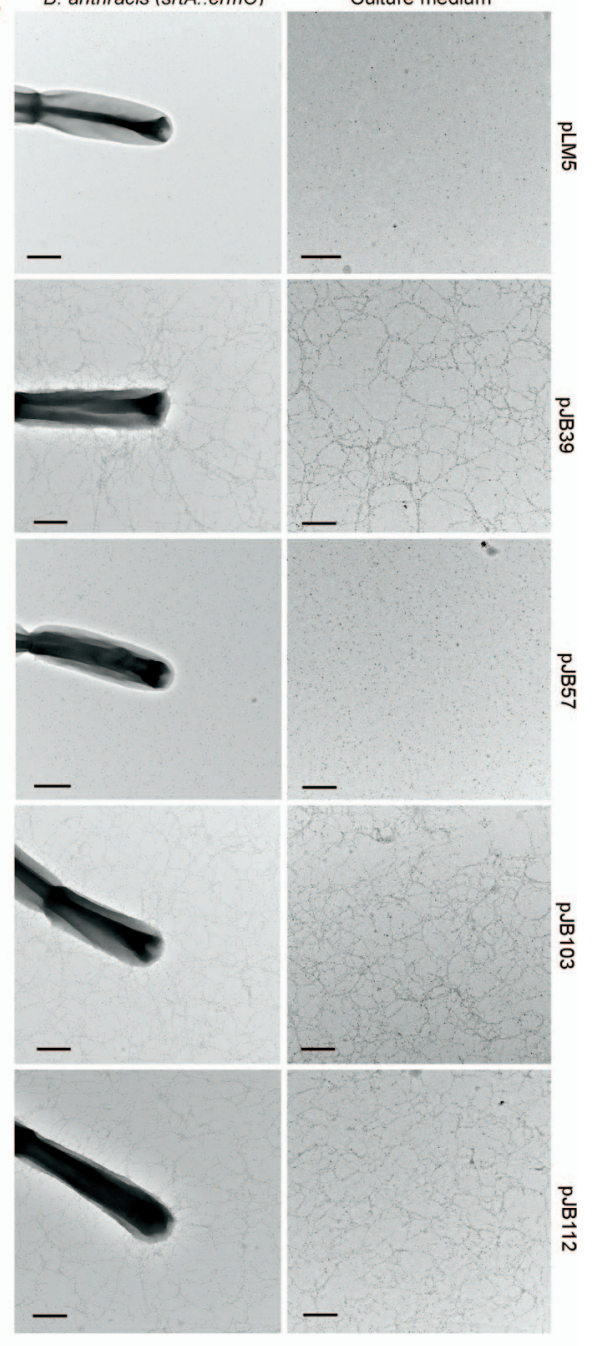

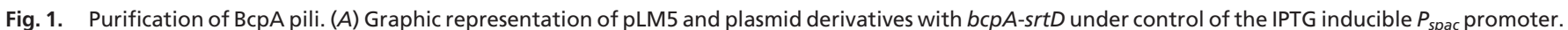

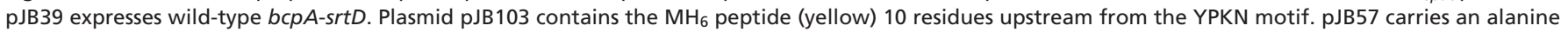

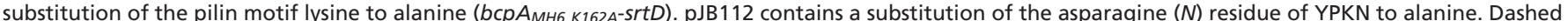

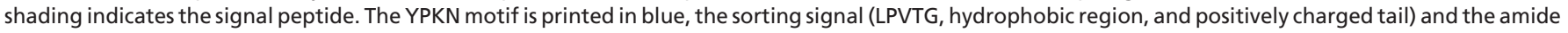

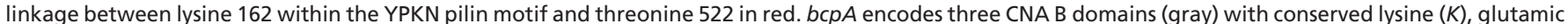

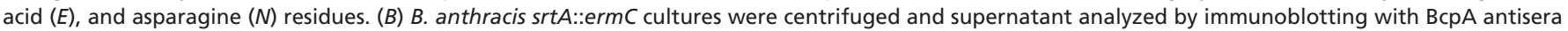

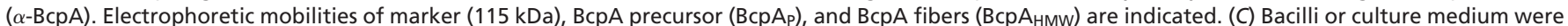

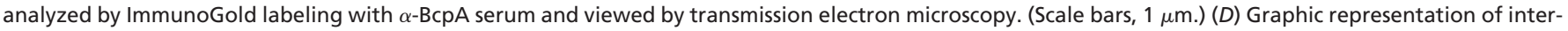

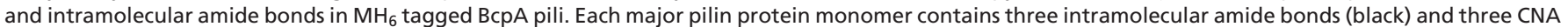

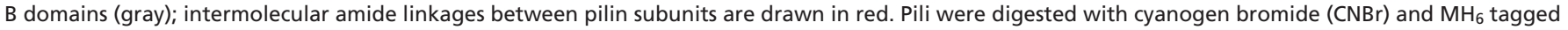
YPKN peptides purified on NI-NTA, separated by SDS/PAGE and stained with Coomassie. Four 500- $\mu$ l eluate aliquots were collected (E1-4).

supporting information (SI) Table S1]. The structure of compound $\mathrm{A}$ is explained as three linear $\mathrm{CNBr}$ cleavage products linked by two amide bonds (Table S2). The N-terminal $\mathrm{CNBr}$ fragment, $\mathrm{Asp}^{26}-\mathrm{Met}^{96}$, is linked to the third $\mathrm{CNBr}$ fragment, 6 His-Gly ${ }^{150}$-Met $^{166}$. The C-terminal (fifth) fragment, Leu ${ }^{519}$ $\mathrm{Thr}^{522}$, is tethered to the third fragment, indicating that during pilus assembly the LPXTG sorting signal is indeed cleaved between the threonine and glycine residues. Electrospray ionization Fourier transform mass spectrometry (ESI-FTMS) identified two species for compound A, $M_{\mathrm{r}} 10,824.59$ and $M_{\mathrm{r}}$ $10,841.59$; the latter is thought to contain homoseryl instead of homoserine lactone, which is acquired during $\mathrm{CNBr}$ cleavage at Met residues (Fig. 2B). A structural model for compound A (predicted average mass $10,825.16 M_{\mathrm{r}}$ ) is displayed in Fig. $2 B$. The carboxyl group of $\mathrm{Thr}^{522}$ (LPXT) is tethered to the sidechain $\varepsilon$-amino group of Lys ${ }^{162}$ (YPKN). A second amide bond, positioned between Asn $^{163}$ (YPKN) and Lys ${ }^{37}$, joins the first and third CNBr fragments. Compound A $\left(10,824.59 M_{\mathrm{r}}\right)$ was fragmented by collision-activated dissociation (CAD) and electron capture dissociation (ECD) and analyzed by ESI-FTMS/MS. Fragment ion spectra were in agreement with the proposed structure (data not shown). Mass spectrometry of compound B revealed that the methionine residues bordering the second fragment had not been cleaved by $\mathrm{CNBr}$, generating essentially the same structure and amide bonds as compound A. Compound $\mathrm{C}$ appears also to be generated by incomplete cleavage and includes the fourth BcpA fragment $\left(38,848.22 M_{\mathrm{r}}\right.$ ) (data not shown). In summary, affinity chromatography and mass spectrometry of YPKN motif peptides released by $\mathrm{CNBr}$ cleavage from BcpA pili revealed two amide bonds, an intermolecular link between the $\mathrm{C}$-terminal threonine and the lysine of the YPKN motif (Lys ${ }^{162}-\mathrm{Thr}^{522}$ ), which is derived by sortase $\mathrm{D}$ cleavage at 

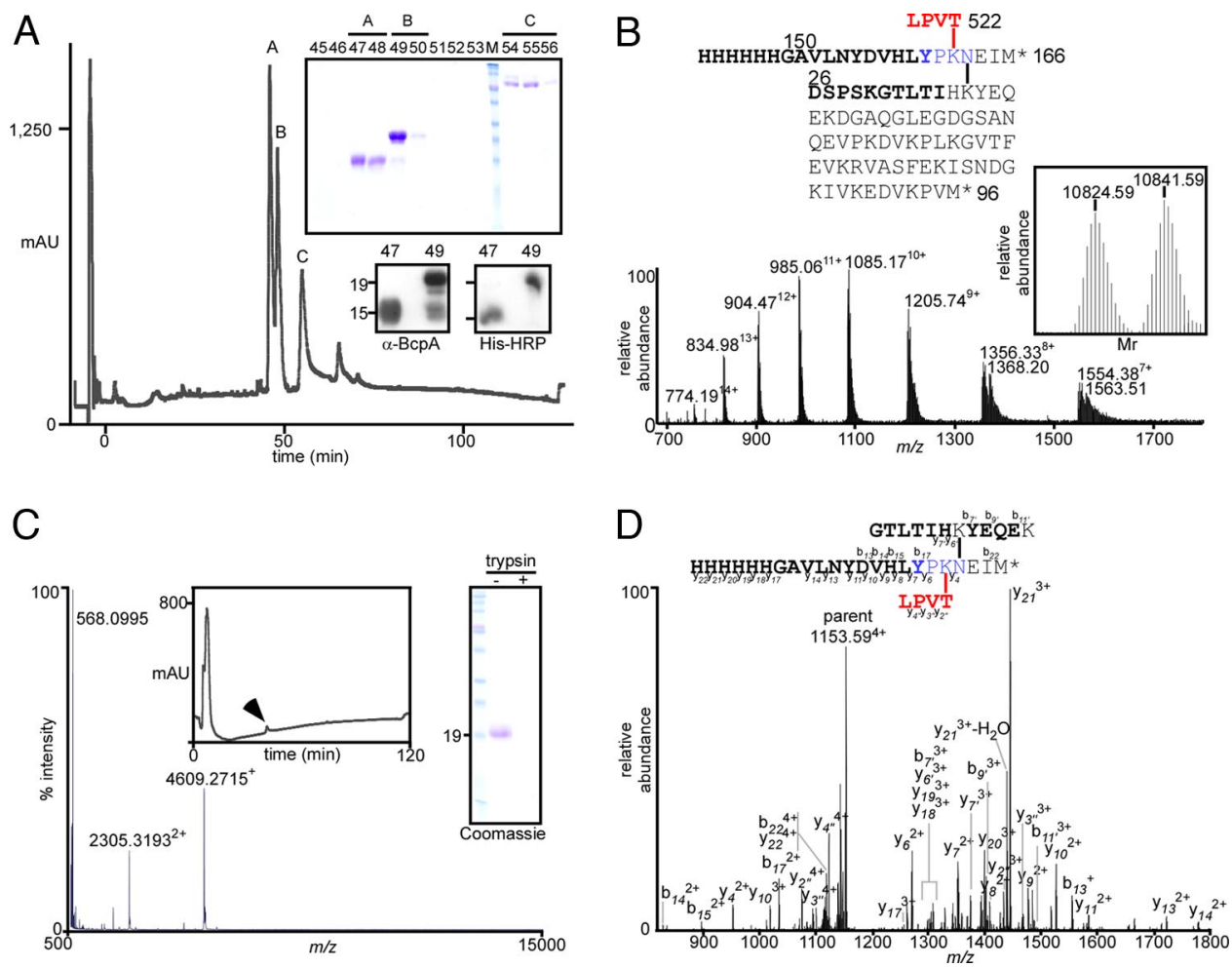

Fig. 2. Inter- and intramolecular amide bonds in BcpA pili. (A) RP-HPLC of CNBr cleaved and Ni-NTA purified BcpA pili. Compounds A-C were analyzed by SDS/PAGE (Coomassie), by immunoblotting with $\alpha$-BcpA and by binding to His-HRP. (B) The molecular mass of compound A was determined by ESI-FTMS. Insets display the deduced structure of compound A and the Mr values calculated by XtractAll for Qual Browser. Intra- (Asn 163-Lys ${ }^{37}$, black), intermolecular amide (Lys ${ }^{162}$-Thr ${ }^{522}$, red), YPKN motif (blue) and LPXTG sorting signal (red) are indicated. $\mathrm{M}^{*}$ denotes homoserine lactone residues. Residues in bold were identified by Edman degradation. (C) Compound A was incubated with trypsin and analyzed by SDS/PAGE (Coomassie). Compound A1, a tryptic peptide of compound A, was purified by Ni-NTA affinity chromatography, separated by RP-HPLC (arrowhead) and mass measured by MALDI-MS. (D) CAD fragmentation spectra (m/z) of the parent ion 1,153.594+. Fragment ions are labeled,' refers to fragment ions that arose from fragmentation of the GTLTIHKYEQEK branch of the peptide, " refers to fragment ions that arose from the LPVT branch of the peptide. Unmarked fragment ions were derived from fragmentation of the HHHGAVLNYDVHLYPKNEIM*. $M *$ denotes a homoseryl residue.

the LPXTG sorting signal (see below), and the intramolecular bond $\mathrm{Lys}^{37}-\mathrm{Asn}^{163}$ in the first CNA B domain of BcpA.

Inter- and Intramolecular Amide Bonds of BcpA. To reveal the amide bonds in compound A by mass spectrometry, YPKN motif peptides were treated with trypsin and purified by Ni-NTA affinity chromatography followed by RP-HPLC. MALDI- and ESI-MS revealed one major compound A1, $m / z$ 4,609.27 and $m / z$ 4609.34, respectively (Fig. $2 C$ and Fig. S1), Edman degradation of which released three residues per cycle (Fig. $2 D$; identified residues printed in bold; Table S1). Fragment ion spectra of $\mathrm{m} / \mathrm{z}$ 4,609.34 were generated in four different modes that together revealed the complete structure of compound A1 (Fig. 2D, Fig. $\mathrm{S} 1$, and Table S2). The Lys ${ }^{162}-\mathrm{Thr}^{522}$ intermolecular amide bond was revealed with the $\mathrm{b}_{19}$ and $\mathrm{a}_{18}-\mathrm{NH}_{3}$ ions fragment ions (Table $\mathrm{S} 2$ ), and the intramolecular amide linkage was revealed with the $\mathrm{y}_{4}$ ion (Table S2).

Intramolecular Amide Bonds Contribute to Pilus Assembly. Many surface proteins of Gram-positive bacteria, including pilin precursors, harbor CNA B domains with conserved lysine and asparagine residues (17). The conserved glutamic acid in CNA $\mathrm{B}$ domains is thought to promote ammonium elimination and Lys-Asn amide bond formation (15). We examined a possible contribution of intramolecular amide bonds toward pilus assembly. $\mathrm{BcpA}_{\mathrm{His}-6}$ (residues 26-522 fused to the C-terminal His tag in pET24b) was purified from Escherichia coli and subjected to Edman degradation and RP-HPLC and ESI-FTMS (Fig. $3 A$ and $B$, Table S3). The calculated average mass of $\mathrm{BcpA}_{\text {His-6 }}$ (without its N-terminal methionine) is $56,697.47$, whereas the measured average mass is $56,648.60$ (Fig. $3 B$ ). The observed difference is explained as the loss of one ammonium ion $(17 \mathrm{Da})$ for each of the three CNA B domains of BcpA. In agreement with this model, substitution of catalytic glutamic acid residues, Glu ${ }^{223}$ or $\mathrm{Glu}^{472}$, with alanine-generated mass differences equivalent to two ammonium ions with respect to the calculated mass, whereas substitution of $\mathrm{Glu}^{223}$ and $\mathrm{Glu}^{472}$ produced a mass difference of one ammonium ion (16.85) (Table S3). Purified BcpA $\mathrm{A}_{\text {His-6 }}$ displayed resistance to trypsin cleavage, and this could be diminished by alanine substitutions at the catalytic glutamic acid residues (Fig. $3 A$ ). B. anthracis was transformed with plasmids pJB12 (bcpA-srtD-bcpB), pJB107 (bcp $A_{E 472 A}$-srtD-bcpB), pJB108 (bcp $A_{E 223 A^{-}}$srtD-bcpB), pJB109 (bcp $\left.A_{N 163 A}-s r t D-b c p B\right)$, or pJB123 (bcp $A_{E 223 A} E 472 A$-srtD-bcpB) and pilus formation examined by electron microscopy and immunoblotting (Fig. $3 C$ and $D$ ). Although alanine substitution of $\mathrm{Glu}^{472}$ alone did not affect pilus assembly, substitutions at $\mathrm{Glu}^{223}$ or $\mathrm{Glu}^{223}$ and $\mathrm{Glu}^{472}$ abolished fiber formation (Fig. 3C). Alanine substitution at $\mathrm{Asn}^{163}$, the YPKN motif residue tethered via amide to Lys ${ }^{37}$, also had no effect on pilus assembly (Fig. 3D). To ascertain that introduction of $\mathrm{Ala}^{163}$ abrogated amide bond formation, pili were cleaved with $\mathrm{CNBr}$, purified by Ni-NTA, and analyzed by RP-HPLC, which revealed four compounds. The mass of the smallest compound $\mathrm{A}^{*}$ was determined as $m / z$ 3,117.76 (Fig. 3E). The structure of compound $\mathrm{A}^{*}$ was characterized by Edman degradation and CAD fragmentation, revealing two linear peptides with one intermolecular amide bond (Lys ${ }^{162}-\mathrm{Thr}^{522}$ ), but without an intramolecular peptide bond (Fig. $3 F$, Table S4). 
A

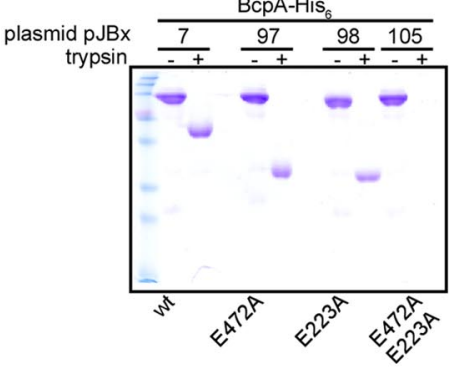

B

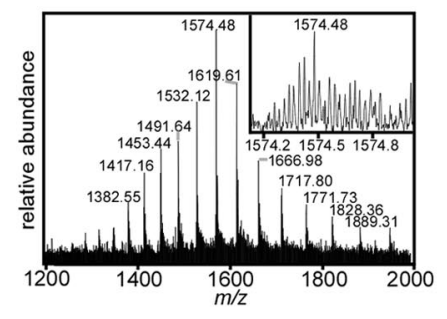

C

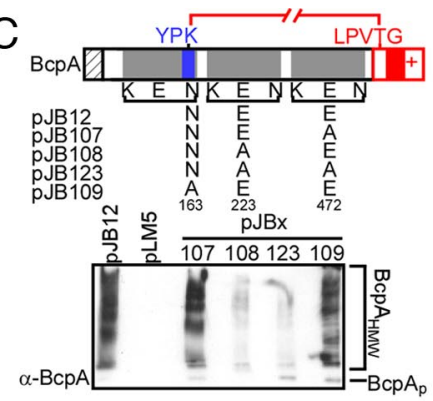

D

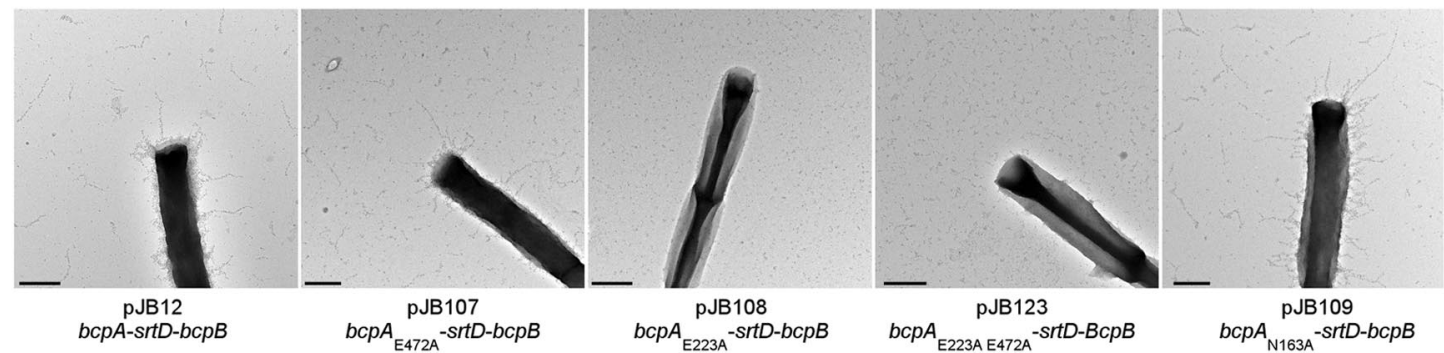

$E$

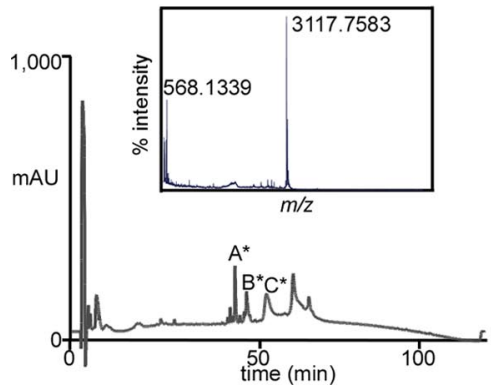

$\mathrm{F}$

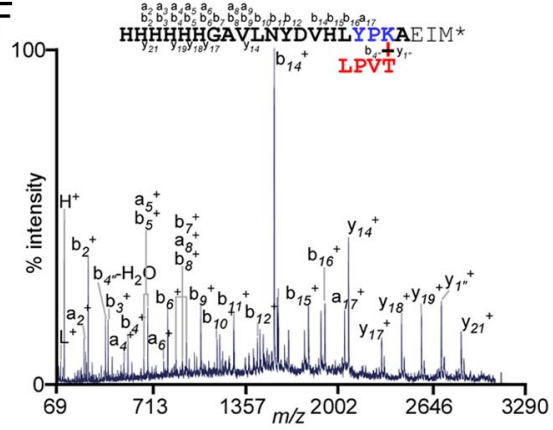

$\mathrm{G}$

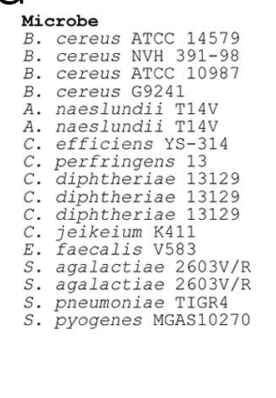

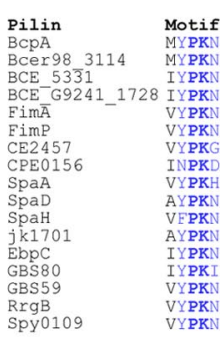

Spy0109

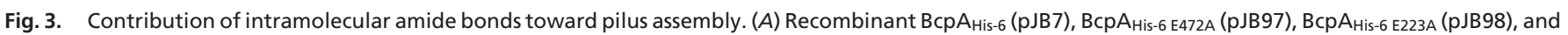

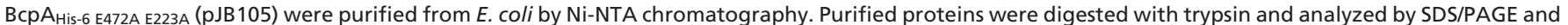

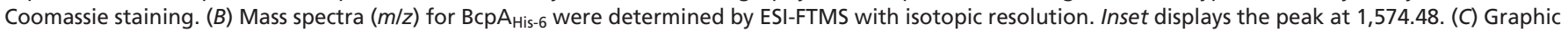

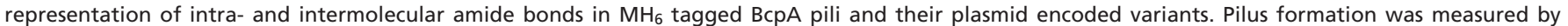

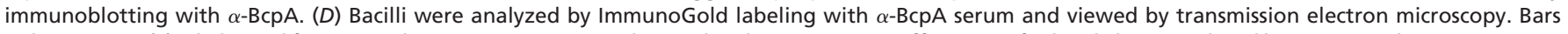

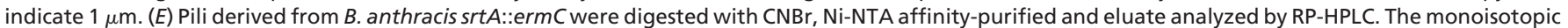

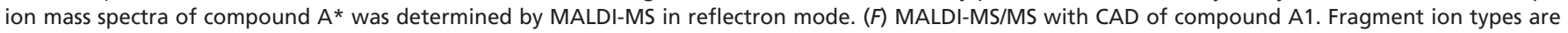

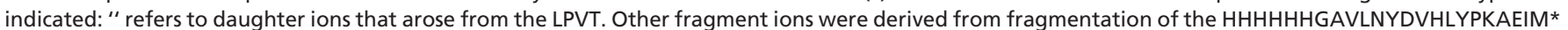

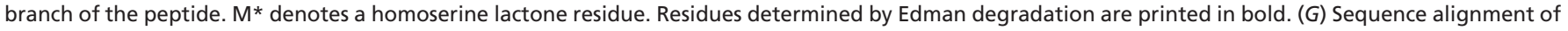
the YPKN motif in major pilin proteins of Gram-positive bacteria.

Sortase A and D Cleave the LPXTG Sorting Signal of BcpA. We sought to identify the enzyme that cleaves LPXTG sorting signals and forms the amide bond (Lys ${ }^{162}-\mathrm{Thr}^{522}$ ) between pilin subunits. Plasmid pJB69 harbors a translational fusion between $b c p A$ and gst, encoding GST tethered to the C-terminal end of the LPXTG sorting signal (Fig. 4A). B. anthracis (pJB69) formed pili on the bacterial surface, whereas $B$. anthracis (pJB86), a plasmid variant lacking sortase D (srtD), did not (Fig. $4 B$ and $C$ ). Bacilli lacking $s r t A$ and $s r t D$ accumulated BcpA-GST precursor but no cleavage products (Fig. 4B). Thus, both sortases cleave BcpA-GST substrate; however, only one of the two transpeptidases, SrtD, polymerizes pili. BcpA-GST and its cleavage products were purified by affinity chromatography on glutathione Sepharose and analyzed by Coomassie-stained SDS/PAGE or immunoblotting with antibodies against BcpA or GST (Fig. $4 D$ and $E$ ). In the absence of $s r t A$ and $s r t D$, bacilli accumulated BcpA-GST pilin precursor with uncleaved LPXTG sorting signal (calculated $M_{\mathrm{r}} 85,094.57$ ) (Fig. $4 D$ and $E$ ). In cells expressing $s r t A$ and/or $s r t D$, BcpA-GST cleavage products were purified (Fig. 4D). Sortase A or D generated cleavage products were recognized by
GST but not by BcpA antisera (Fig. 4E). Cleavage products (M) were subjected to Edman degradation (Fig. 4D). For both SrtAand SrtD-derived BcpA-Gst products, the cleavage site was the glycine of the LPVTG sorting signal, because all subsequent residues agreed with the predicted downstream sequence (Fig. $4 D$ and Table S1).

\section{Discussion}

Assembly of pili in the envelope of $B$. anthracis requires two sortases, SrtA and SrtD (12). Mutations in srtD, either deletion of the ORF or alanine substitution of the active site $\left(\mathrm{Cys}^{207}\right)$, abrogate pilus assembly (12). Mutations in $s r t A$ prevent proper deposition of pili in the envelope, because mutant bacilli release elongated pilus fibers into the extracellular medium (12). In $S$. aureus, sortase A cleaves LPXTG motif sorting signals and forms an amide bond between the $\mathrm{C}$-terminal threonine and the amino group within lipid II $(18,19)$, i.e., the precursor of peptidoglycan synthesis (20). Using a similar mechanism, B. anthracis sortase A is thought to link pili to peptidoglycan cross-bridges in the cell-wall envelope $(12,21)$. This model is corroborated by studies 
A
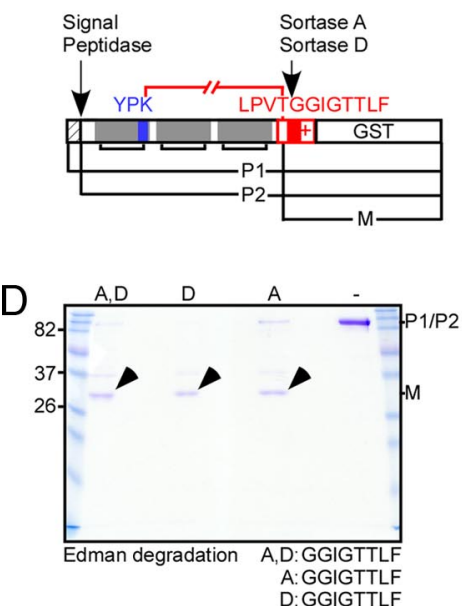

$\mathrm{B}$

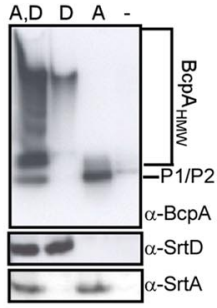

E

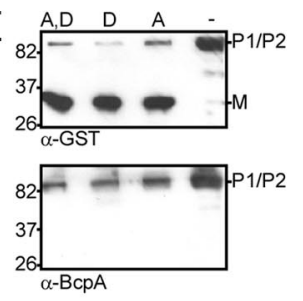

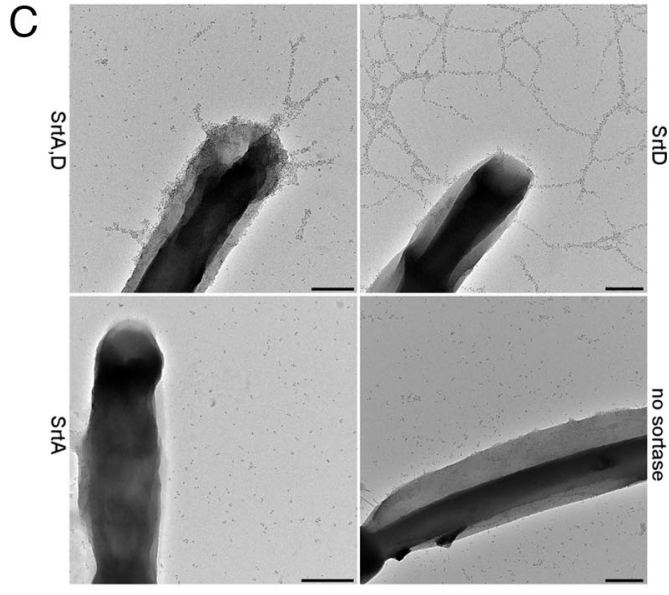

Fig. 4. Cleavage sites of sortases and their contribution to pilus assembly. (A) Diagram displays the precursor (P1) of BcpA-GST, and the signal peptidase (P2) and sortase cleaved products (M). (B) Pilus formation from BcpA-GST substrate was examined by immunoblotting with $\alpha$-BcpA in cells harboring both sortase $\mathrm{D}(\mathrm{SrtD})$ and sortase A (SrtA), only one of the two sortases or none at all. (C) Bacilli were analyzed by ImmunoGold labeling with $\alpha$-BcpA serum and viewed by transmission electron microscopy. (Scale bars, $1 \mu \mathrm{m}$.) $(D)$ The cell wall of bacilli was removed with PlyL, protoplast lysed by sonication, and sortase cleavage products purified by affinity chromatography on glutathione-Sepharose. Electrophoretic mobility of the molecular weight marker, pilin precursors (P1/P2), and mature cleavage products (M) on SDS/PAGE is indicated. Edman degradation revealed the N-terminal sequences of mature products. (E) Purified cleavage products were subjected to immunoblotting with specific antisera ( $\alpha$-GST or $\alpha$-BcpA).

in $C$. diphtheriae and $S$. agalactiae, where $s r t A$ mutants cause similar phenotypes $(22,23)$. We show that SrtA and SrtD cleave pilin precursor at the same site, the LPXTG sorting signal; however, only SrtD promotes pilus assembly. Thus, SrtA cleavage of pilin precursor that is already attached to assembled BcpA fibers terminates pilus assembly by removal of the sorting signal.

BcpA cleavage by SrtD leads to the assembly of pili, fibrous structures formed by intermolecular amide bonds between the C-terminal threonine of cleaved sorting signals $\left(\mathrm{Thr}^{522}\right)$ and the conserved lysine residue of the YPKN motif (Lys ${ }^{162}$ ). The acyl enzyme of SrtD can, therefore, be viewed as an assembly platform for the capture of polymerized pili via thioester linked intermediates with the C-terminal threonine of the last pilin subunit. The $\varepsilon$-amino group of $\mathrm{Lys}^{162}$ in $\mathrm{BcpA}$ pilin precursor completes the transpeptidation reaction; however, the product again serves as a catalytic substrate when SrtD cleaves its LPXTG sorting signal. BcpB, the tip protein of BcpA pili, also harbors a sorting signal (IPNTG) and is substrate for SrtD and SrtA cleavage (12). Because BcpB lacks the YPKN motif, $\mathrm{BcpB}-\mathrm{SrtD}$ intermediates can only be resolved by the BcpA nucleophile (Lys ${ }^{162}$ ), thereby positioning $\mathrm{BcpB}$ at the tip of polymerizing BcpA fibers. Kang et al. (15) reported an amide bond between the C-terminal threonine ( $\mathrm{Thr}^{311}$ ) and lysine 161 $\left(\right.$ Lys $\left.^{161}\right)$ of Spy0128. Unlike other major pilin proteins of Grampositive bacteria, most Group A streptococcal pilins do not harbor the YPKN motif $(3,24)$. The contribution of Lys ${ }^{161}$ or any other lysine residue toward streptococcal pilus assembly remains unknown (15).

BcpA harbors three CNA B domains with intramolecular amide bonds. These bonds likely stabilize the Ig-fold of CNA B domains (15) and provide resistance to protease, as is characteristic for pili of Gram-positive bacteria $(3,25)$. Nevertheless, only the second amide bond of BcpA is required for pilus assembly, whereas the bond between Lys ${ }^{37}-\mathrm{Asn}^{163}$, immediately adjacent to the YPKN motif nucleophile, is dispensable. The asparagine of the YPKN motif is not absolutely conserved in pilin proteins, and intramolecular amide bonds at this site may be generally dispensable for pilus assembly (Fig. $3 G$ ). We surmise that, apart from incorporating peptidase resistance, intramolecular amide bonds may be important for precursor folding and presentation of the YPKN nucleophile to SrtD intermediates. Conservation of sortase catalysts and pilin proteins with the YPKN motif, the LPXTG sorting signal, and the CNA B domains suggests that the mechanism of pilus assembly is universal in Gram-positive bacteria (26). These insights may aid in the design of pilus-derived vaccines to prevent diseases caused by Gram-positive bacteria (27).

\section{Materials and Methods}

Bacterial Strains. Coding sequences of the $b c p A-s r t D-b c p B$ operon were PCR amplified and cloned into pLM5 (28), to generate IPTG-inducible expression via the $\mathrm{P}_{\text {spac }}$ promoter in $B$. anthracis. Oligonucleotides were used to create single amino acid substitutions in $b c p A$ by site-directed mutagenesis (Table S5). pJB7 (BcpA-His $s_{6}$ in pET24-b) (12) was used as template to derive substitution mutations via Quick-Change mutagenesis.

Pilus Assembly. B. anthracis Sterne or an isogenic $\Delta(s r t A)$ mutant harboring plasmids were grown overnight at $30^{\circ} \mathrm{C}$ on LB agar with kanamycin and IPTG. Cells were suspended in $100 \mathrm{mM} \mathrm{NaCl}$ and analyzed immunoelectron microscopy with $\alpha$-BcpA antisera and 10-nm gold anti-rabbit IgG conjugate (12). For immunoblotting, supernatant aliquots or mutanolysin-digested bacillus extracts were precipitated with TCA, separated by SDS/PAGE, electrotransferred to the PVDF membrane, and antibody binding was revealed by chemiluminescence. For BcpAGST analysis, bacilli were digested with PlyL. Sonicated protoplast extracts were subjected to glutathione Sepharose chromatography and eluates analyzed by SDS/PAGE, immunoblotting, ESI-MS/MS, or Edman degradation.

Analysis of Purified Pili. Bacilli were grown on LB agar, suspended in water, and centrifuged and pili in the supernatant were digested with $\mathrm{CNBr}$ (29). Reactions were dried, solubilized and peptides chromatographed on Ni-NTA Sepharose. Eluate was subjected to RP-HPLC $\left(C_{8}\right)$ and peptides analyzed by SDS/PAGE, mass spectrometry, Edman degradation, and trypsin cleavage.

Mass Spectrometry. Peptides were cospotted with matrix ( $\alpha$-cyano-4hydroxycinnamic acid) and subjected to MALDI-MS on a TOF instrument in linear and reflectron modes. For ESI-FTMS, compounds were individually loaded into nanospray emitters, desorbed by spray voltage between 1.2 and $1.4 \mathrm{kV}$, and analyzed in a hybrid linear ion-trap/FTICR mass spectrometer. Fragmentation experiments included CAD, ECD, aiECD, and IRMPD.

Bacterial Strains, Pilus Purification and Mass Spectrometry. Detailed methods are described in SI Text. 
ACKNOWLEDGMENTS. This work was funded by United States Public Health Service Grants Al38897 and Al69227 (to O.S.). J.M.B was a trainee of the National Institutes of Health Medical Scientist Training Program at University of Chicago (GM07281). L.A.M. was supported by the William Rainey Harper

1. Barocchi MA, et al. (2006) A pneumococcal pilus influences virulence and host inflammatory responses. Proc Natl Acad Sci USA 103:2857-2862.

2. Lauer P et al. (2005) Genome analysis reveals pili in Group B streptococcus. Science 309:105.

3. Mora M et al. (2005) Group A Streptococcus produce pilus-like structures containing protective antigens and Lancefield T antigens. Proc Natl Acad Sci USA 102:1564115646

4. Nelson AL et al. (2007) RrgA is a pilus-associated adhesin in Streptococcus pneumoniae. Mol Microbiol 66:329-340.

5. Yeung MK, Cisar JO (1988) Cloning and nucleotide sequence of a gene for Actinomyces naes/undii WVU45 type 2 fimbriae. J Bacteriol 170:3803-3809.

6. Maione $D$ et al. (2005) Identification of a universal group $B$ streptococcus vaccine by multiple genome screen. Science 309:148-150.

7. Gianfaldoni $C$, et al. (2007) Streptococcus pneumoniae pilus subunits protect mice against lethal challenge. Infect Immun 75:1059-1062.

8. Ton-That $\mathrm{H}$, Schneewind $\mathrm{O}$ (2003) Assembly of pili on the surface of $\mathrm{C}$ diphtheriae. $\mathrm{Mol}$ Microbio/ 50:1429-1438.

9. Mazmanian SK, Liu G, Ton-That H, Schneewind O (1999) Staphylococcus aureus sortase, an enzyme that anchors surface proteins to the cell wall. Science 285:760-763.

10. Ton-That H, Liu G, Mazmanian SK, Faull KF, Schneewind O (1999) Purification and characterization of sortase, the transpeptidase that cleaves surface proteins of Staphylococcus aureus at the LPXTG motif. Proc Natl Acad Sci USA 96:12424-12429.

11. Mishra A, Das A, Cisar JO, Ton-That H (2007) Sortase-catalyzed assembly of distinct heteromeric fimbriae in Actinomyces naes/undii. J Bacteriol 189:3156-3165.

12. Budzik JM, Marraffini LA, Schneewind O (2007) Assembly of pili on the surface of Bacillus cereus vegetative cells. Mol Microbiol 66:495-510.

13. Ton-That $H$, Marraffini LA, Schneewind $O$ (2004) Sortases and pilin elements involved in pilus assembly of Corynebacterium diphtheriae. Mol Microbiol 53:1147-1156.

14. Schneewind O, Fowler A, Faull KF (1995) Structure of the cell wall anchor of surface proteins in Staphylococcus aureus. Science 268:103-106.

15. Kang HJ, Coulibaly F, Clow F, Proft T, Baker EN (2007) Stabilizing isopeptide bonds revealed in gram-positive bacterial pilus structure. Science 318:1625-1628.
Fellowship of University of Chicago. O.S. acknowledges membership within and support from the Region V "Great Lakes" Regional Center of Excellence in Biodefense and Emerging Infectious Diseases Consortium (GLRCE, Nationa Institute of Allergy and Infectious Diseases Award 1-U54-AI-057153).

16. Krishnan V, et al. (2007) An IgG-like domain in the minor pilin GBS52 of Streptococcus agalactiae mediates lung epithelial cell adhesion. Structure 15:893-903.

17. Deivanayagam CC, et al. (2000) Novel fold and assembly of the repetitive B region of the Staphylococcus aureus collagen-binding surface protein. Structure 8:67-78.

18. Ton-That H, Mazmanian H, Faull KF, Schneewind O (2000) Anchoring of surface proteins to the cell wall of Staphylococcus aureus. J Biol Chem 275:9876-9881.

19. Perry AM, Ton-That H, Mazmanian SK, Schneewind O (2002) Anchoring of surface proteins to the cell wall of Staphylococcus aureus. J Biol Chem 277:16241-16248.

20. Higashi Y, Strominger JL, Sweeley CC (1967) Structure of a lipid intermediate in cell wall peptidoglycan synthesis: A derivative of C55 isoprenoid alcohol. Proc Natl Acad Sci USA 57:1878-1884

21. Marraffini LA, DeDent AC, Schneewind O (2006) Sortases and the art of anchoring proteins to the envelopes of gram-positive bacteria. Microbiol Mol Biol Rev 70:192221.

22. Swaminathan A, et al. (2007) Housekeeping sortase facilitates the cell wall anchoring of pilus polymers in Corynebacterium diphtheriae. Mol Microbiol 66:961-974.

23. Dramsi S, et al. (2006) Assembly and role of pili in group B streptococci. Mol Microbiol 60:1401-1413.

24. Schneewind O, Jones KF, Fischetti VA (1990) Sequence and structural characteristics of the trypsin-resistant T6 surface protein of group A streptococci. J Bacteriol 172:3310 3317.

25. Lancefield R (1962) Current knowledge of type-specific M antigens of group A streptococci. J Immunol 89:307-313.

26. Ton-That $\mathrm{H}$, Schneewind $\mathrm{O}$ (2004) Assembly of pili in gram-positive bacteria. Trends Microbiol 12:251-261.

27. Telford JL, Barocchi M, Margarit I, Rappuoli R, Grandi G (2006) Pili in gram-positive pathogens. Nat Rev Microbiol 4:509-519.

28. Marraffini LA, Schneewind O (2006) Targeting proteins to the cell wall of sporulating Bacillus anthracis. Mol Microbiol 62:1402-1417.

29. Ton-That H, Faull KF, Schneewind O (1997) Anchor structure of staphylococcal surface proteins. J Biol Chem 272:22285-22292. 